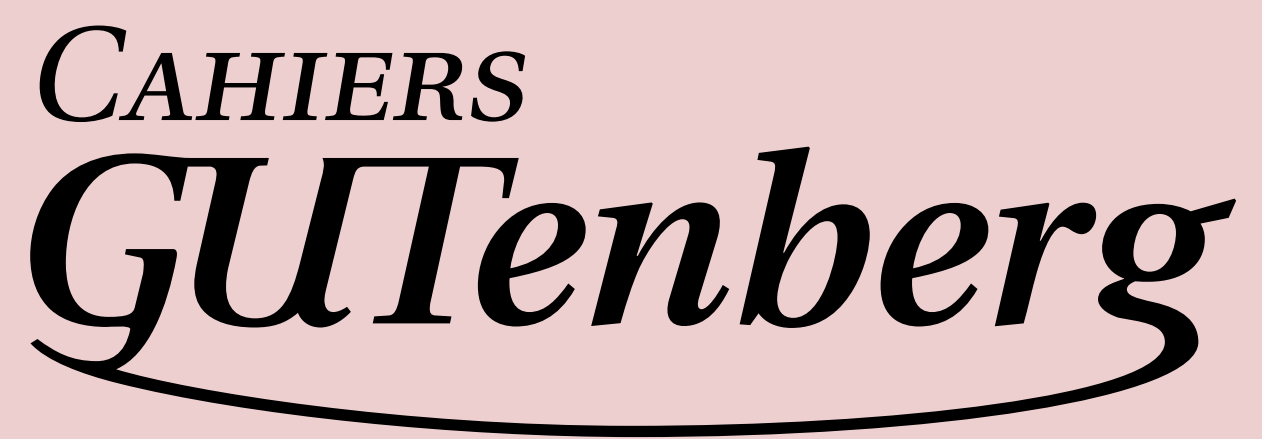

\title{
שु FONTES ET DESSINATEURS DE FONTES HONGROIS CONTEMPORAINS
}

\section{व Gyöngyi BujDosó}

Cahiers GUTenberg, no 46-47 (2006), p. 43-57.

<http://www.gutenberg.eu.org/publications/cahiers/>

๑) Association GUTenberg, 2006, tous droits réservés.

L'accès aux articles de la revue "Cahiers GUTenberg " (http://www.gutenberg.eu.org/publications/cahiers/), implique l'accord avec les conditions générales d'utilisation (http://www.gutenberg.eu.org/publications/22-avertissements.html). Toute utilisation commerciale ou impression systématique est constitutive d'une infraction pénale. Toute copie ou impression de ce fichier doit contenir la présente mention de copyright. 



\section{$\mho^{\circ}$ FONTES}

\section{ET DESSINATEURS DE FONTES HONGROIS CONTEMPORAINS}

\section{đ Gyöngyi BujDosó}

RÉsumé. - On nous demande souvent comment utiliser la fonte Janson sous $\mathrm{T}_{\mathrm{EX}}$. Cette très belle fonte ancienne a été dessinée par un graveur de caractères hongrois, M. Tótfalusi Miklós (Nicolas Kis), au XVII ${ }^{\mathrm{e}}$ siècle. Son nom est bien connu, mais peu de gens connaissent d'autres caractères hongrois, comme Pannon Antikva, Tyrnavia, etc., ni d'autres caractères créés par des Hongrois de talent.

Le but de cet article est de donner un bref aperçu de certains de ces caractères et de préfigurer les fruits potentiellement riches d'une collaboration entre la communauté $\mathrm{T}_{\mathrm{E}} \mathrm{X}$ hongroise et les gens du métier.

Aвstract. - Several people have asked for information on how to use Janson fonts with $\mathrm{T}_{\mathrm{E}} \mathrm{X}$. This wonderful antique font was designed by a Hungarian type designer, M. Tótfalusi Miklós (Nicholas Kis) in the 17th century. His name is well-known, but few people know the other, wonderful types such as Pannon Antikva, Tyrnavia, etc., created by talented Hungarians designers.

The aim of this paper is to give a short overview of some new types by Hungarians type designers, and portray the work and the achievements of cooperation between $\mathrm{T}_{\mathrm{E}} \mathrm{X}$ people and typographers.

Note. - Cet article est paru dans TUGBoAT, volume 24 (2003), n 3 - Eurot $T_{E} 2003$ Proceedings (Yannis Haralambous éd.), sous le titre «Contemporary Hungarian Types and Designers», p. 527-530. Traduction française de Jacques André. Il est reproduit ici avec l'autorisation de l'auteur, de Karl Berry (président de TUG) et de Barbara Beeton (TUGвоAт editor).

Cahiers GUTenberg no 46-47 — avril 2006, p. 43-57. 


\section{Motivations}

On nous demande souvent comment utiliser la fonte Janson sous $\mathrm{T}_{\mathrm{E}} \mathrm{X}$. Cette très belle fonte ancienne a été dessinée par un graveur de caractères hongrois, M. Tótfalusi Miklós (Nicolas Kis). Son nom et son Janson sont bien connus dans la communauté $\mathrm{T}_{\mathrm{E}} \mathrm{X}$. Lors d'une conférence BachoT $_{\mathrm{E}} \mathrm{X}$, on nous a questionné au sujet de Pannon Antikva dessinée par Edit Zignány, ainsi que d'autres fontes dessinées par des créateurs hongrois, et sur leur numérisation. Après avoir écrit divers messages électroniques, surfé sur le web ou téléphoné à diverses maisons de publicité ou d'édition, il nous a semblé qu'apparemment il n'y avait aucun dessinateur de caractères en Hongrie! Pourtant, nous avons de nombreux dessinateurs de caractères talentueux, mais ça prend du temps pour les trouver à cause du manque de contacts entre les utilisateurs de $\mathrm{T}_{\mathrm{E}} \mathrm{X}$ et les dessinateurs.

Nous nous proposons, dans ce bref aperçu, de collecter et montrer quelques beaux exemples et de mentionner le nom de quelques Hongrois dessinateurs de caractères de talent, en donnant des exemples chaque fois que possible. À la fin de cet article, nous donnerons quelques nouvelles sur l'implantation de $\mathrm{T}_{\mathrm{E}} \mathrm{X}$ en Hongrie.

\section{QUELQUES BELLES FONTES}

Quand on parle de fontes hongroises, on ne peut pas oublier les précurseurs qui ont influencé le dessin des fontes contemporaines.

La fameuse fonte connue sous le nom de Janson est l'œuvre de Nicolas Kis (1650-1702). Le nom hongrois de Nicolas Kis est M. Tótfalusi (ou Misztótfalusi) Kis Miklós. Il a fait ce caractère, ainsi que d'autres, durant un séjour à Amsterdam (1680-1688) où il avait été envoyé pour publier une nouvelle version hongroise de la Bible. La figure 1 montre la couverture de cette seconde édition hongroise, modernisée et corrigée, de la Bible.

Après quelques mois d'études, il commença à graver des fontes complexes et à réparer des matrices défectueuses. Le Janson qui en a résulté est bien connu (figure 2). Le nom de Janson est en fait erroné ; on a longtemps cru que ce caractère avait été dessiné par le dessinateur danois Anton Janson qui a été fondeur à Leipzig durant les années 1668-1687. Cette fonte datant du Baroque est adaptée à toutes sortes d'usages. Les utilisateurs de $\mathrm{T}_{\mathrm{E}} \mathrm{X}$ peuvent employer les fontes PostScript Janson Text 


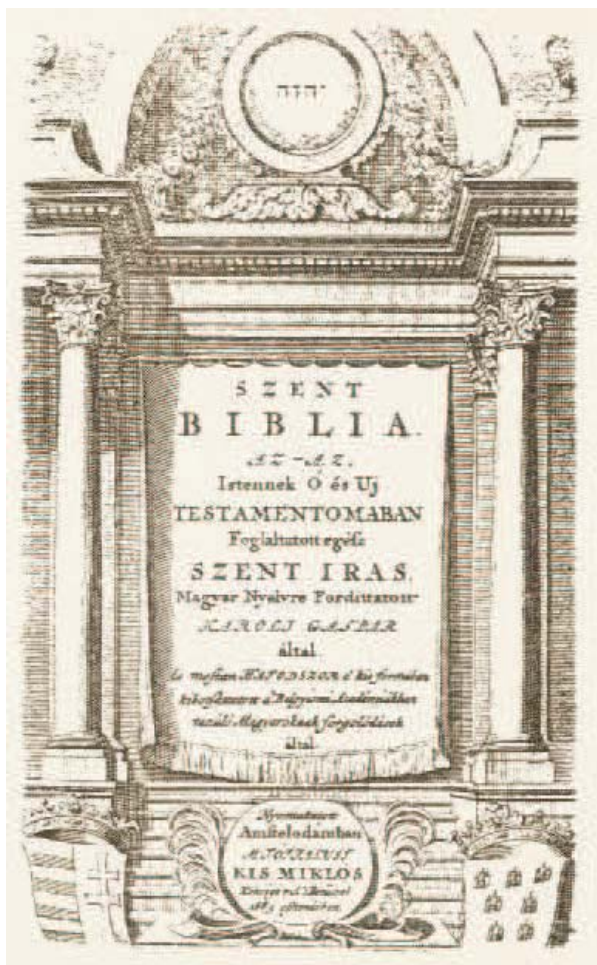

Figure 1. - Bible traduite en hongrois, éditée, modernisée et corrigée par Nicolas Kis. Image extraite de [15].

\section{ABCDEFGHIJKL ABCDEFGHIFKL MNOPQRSTUVW MNOPQRSTUVW XYZabcdefghijklmn XYZabcdefghijklmn opqrstuvwxyz\&0123 opqrstuvwxyz\&0123

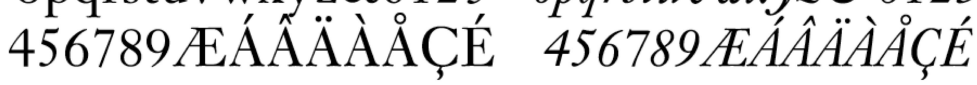

Figure 2. - Spécimen du Janson Text d'Adobe [16].

Fontes et dessinateurs de fontes hongrois contemporains 


\section{balaton 99}

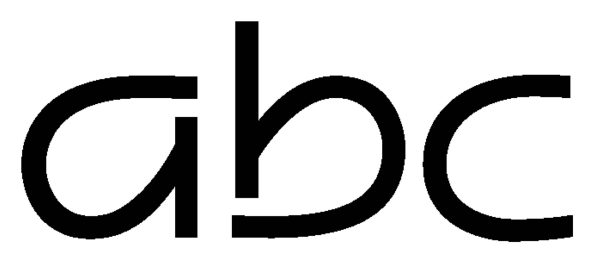

Figure 3. - Balaton de László Bujáki.

d'Adobe [16], de Corel et de Monotye. Il existe d'autres polices influencées directement des fontes de Nicolas Kis, comme les Stempel Janson, Mergenthaler Linotype Janson et Bistream Kis [6].

En ce qui concerne la typographie du $\mathrm{xx}^{\mathrm{e}}$ siècle, nous devons tout d'abord citer le caractère Margaret dessiné par Zoltán Nagy (1920-1998) qui gagna le troisième prix du concours ITC à New-York. Cette fonte est utilisée pour les passeports hongrois [18]. Oszkár Boskowitz a numérisé les caractères de Z. Nagy et les a implémentés et modifiés avec l'accord des héritiers du dessinateur.

László Bujáki [2], un élève de Zoltán Nagy, avait commencé à adapter les fontes anglaises aux langues de l'Europe de l'Est en modifiant les accents des lettres accentuées sous la direction de Nagy. Il utilisa son propre caractère Cheri pour composer le livre de Nagy intitulé $A$ betütervezés technikája (Techniques du dessin de caractères), imprimé en douze exemplaires à l'occasion du soixante-dixième anniversaire de son professeur. Malheureusement, ces fontes ont disparu avec l'arrivée de la composition photomécanique. Cependant, l'une de ses fontes, Balaton (figure 3), a été numérisée par l'un de ses élèves dix ans après sa conception. Ce magnifique caractère dorique est excellent pour les titres, les affiches et les logos. Nous attendons son nouveau caractère, appelé Bulaton, qui devrait être disponible bientôt.

Cahiers GUTenberg no 46-47 - avril 2006 


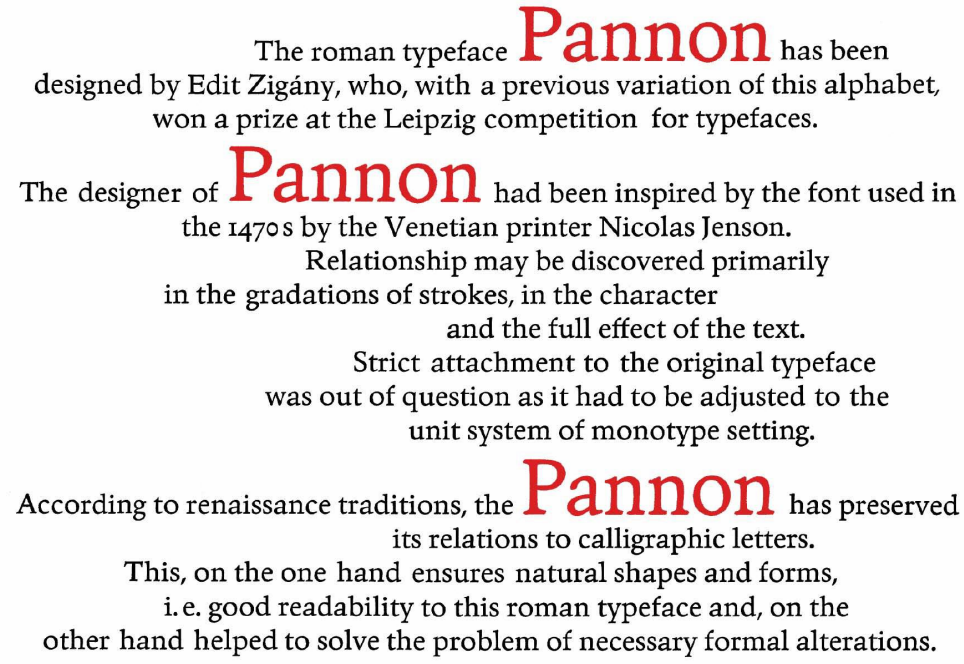

Figure 4. - Pannon d’Edit Zigány.

Le caractère Pannon ${ }^{1}$ (figure 4) dessiné par Edit Zigány ${ }^{2}$ dans les années 1970 est bien connu des typographes. Son tracé esthétique, harmonieux et délicat permet de l'utiliser en édition professionnelle. Il a reçu un (second) prix au concours de caractères de Leipzig. Comme elle n'a pas pu achever Pannon, Oszkár Boskovitz a poursuivi le travail. Il a dessiné les caractères et graisses manquants (gras et petites capitales, en particulier), et a avancé la numérisation de ce caractère.

Oszkár Boskovitz [1] a dessiné quelques polices qui forcent l'admiration, par exemple l'élégante Troppauer, Konwektor et Syrup qui évoquent deux styles différents du siècle dernier, Tilos et Wagon font référence à la culture urbaine, tandis que Thaifun est orientalisante (figure 5).

Il soutient la typographie hongroise. Par exemple, toutes les polices utilisées dans le magazine Off Line, consacré aux sports extrêmes, sont

1. Pannon : Edit Zigány 1972, Oszkár Boskovitz 2001.

2. Edit Zigány est décédée l'année dernière. Elle restera dans notre mémoire. 


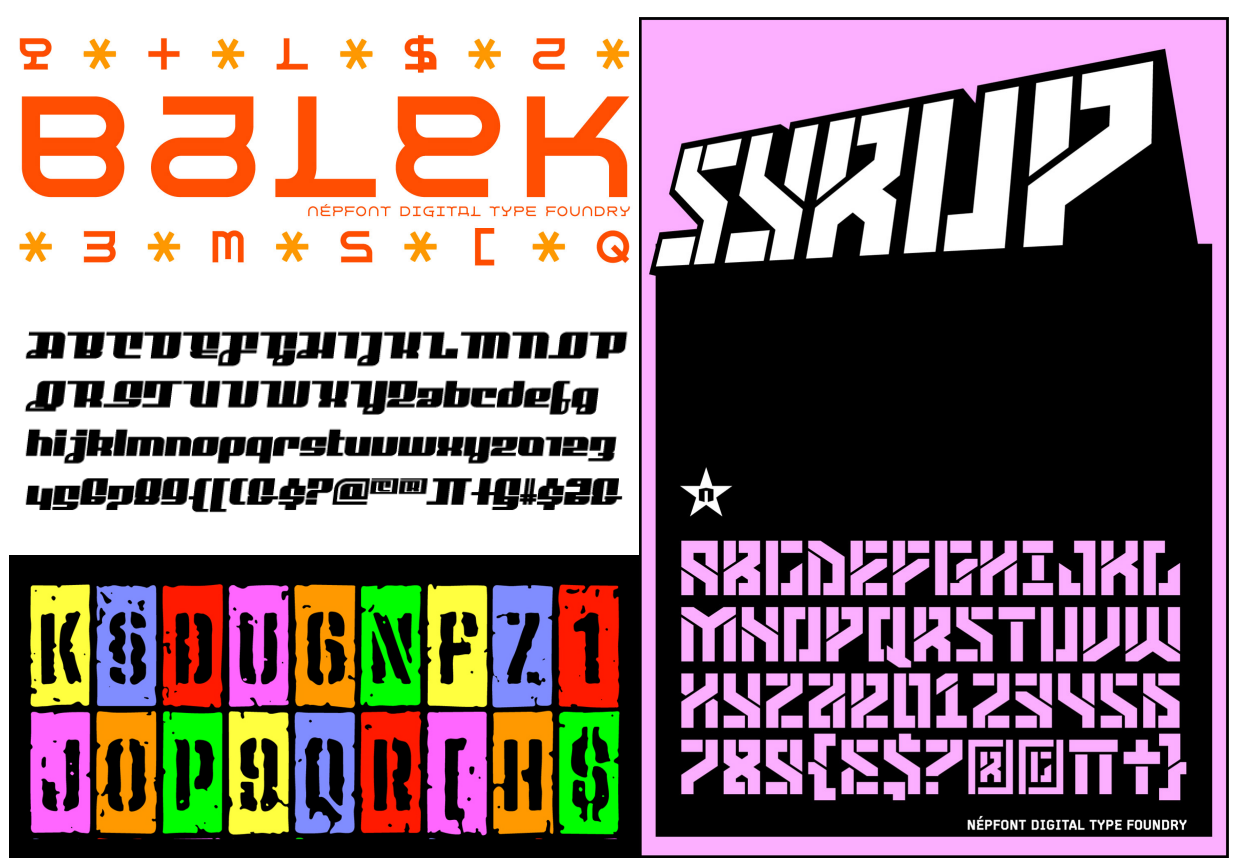

Figure 5. - Balek, Thaifun, Tilos et Syrup d’Oszkár Boskowitz.

d'origine hongroise. Nous attendons de sa part un livre qui va inventorier les caractères dessinés en Hongrie dans les années 1970 et 1980.

György Szőnyei est dessinateur de caractères et graphiste; il a gagné divers prix lors de grands concours. Mentionnons ici les variantes de son Labirinth où chaque lettre est dessinée d'un seul trait, la fonte kanji Möbius et enfin son Transylvanian Script influencé par le travail de Károly Kós, basé sur des épitaphes transylvaniens (figure 6). Son Archian a six variantes [7] dont Plastic et Night sont des jeux en trois dimensions sur la «forme normale» constructiviste.

Péter Csatai [4] produit également des polices inspirées du siècle dernier, comme Fontster et Cube (années 1960-1970), ou Kabos (années 1920-1930). Il a dessiné quelques polices marquantes (Acydum, Antagon...), ainsi que des fontes gracieuses comme Colibry, Pallos ou Hungarum Antiqua (figure 7). Il aime dessiner à partir d'objets purement 
géométriques (Oktan, Apsis), mais il adore les idées étranges comme le développement des machines depuis la révolution industrielle (que l'on retrouve dans Masina).

Grapefruit (figure 8), dessinée par Attila Győry, est une «fonte de titrage anguleuse, maniérée et géométrique avec une forte présence", diffusée depuis 1997 [11]. (Des échantillons d'ITC Grapefruit se trouvent chez Linotype [10].) Attila Győry est maquettiste chez un éditeur. Nous espérons qu'il poursuivra son travail.

Aujourd'hui, Gábor Kóthay et Amondó Szegi sont probablement les dessinateurs de caractères les plus productifs et les plus connus en Hongrie. Ils ont dessiné et réalisé un grand nombre de caractères très réussis, dont le splendide Tyrnavia (figure 9), qui est une tentative de recréation du Kis dessiné à Cluj (Kolozsvár). Ils ont fait des polices destinées à des emplois aussi divers que : texte courant, titraille, affiche, annonce, décoration, carte de vœux, etc., et ce en des styles très variés : sérieux, ludique, archaïque, moderne, manuscrit (plume, brosse, feutre,

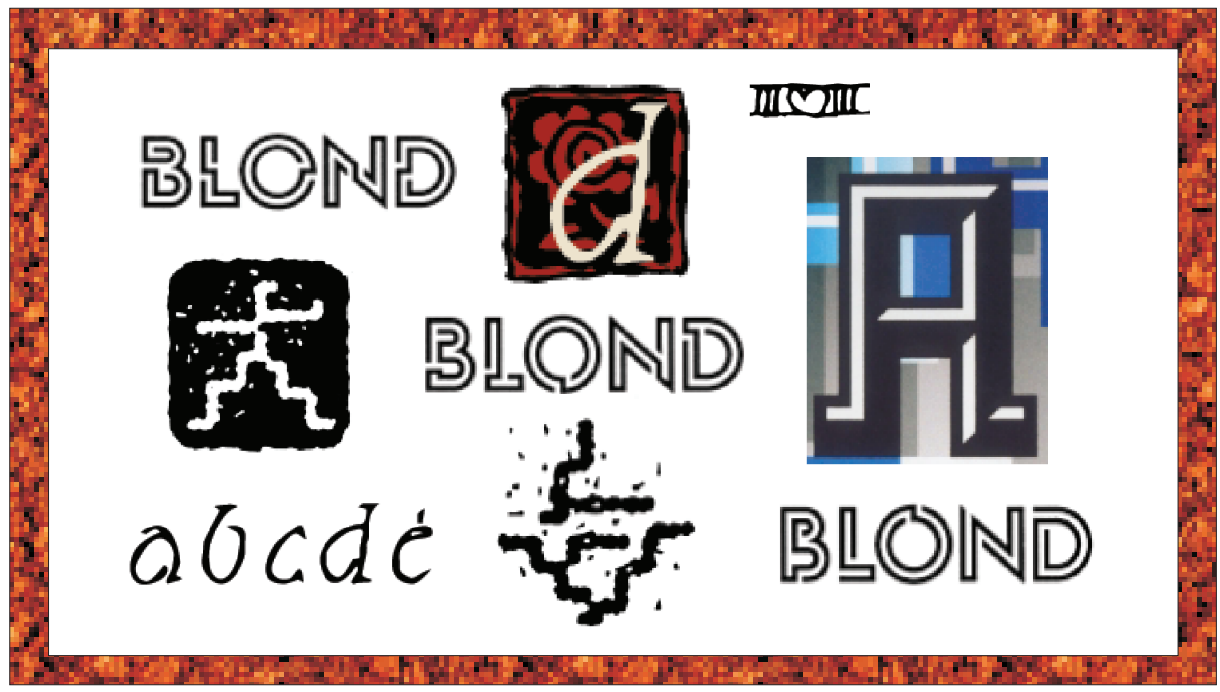

Figure 6. - Labirinth, Möbius, Transylvanian Script, et Archian Plastic de György Szőnyei. 


\begin{tabular}{|c|c|}
\hline masina & colibry \\
\hline ABCDEFGHIJKLMNOPQR & abcdefghisklmnop \\
\hline stuuшк ч zo12345678 g\#\$ & $m n \circ p Q r s t u v W \times Y Z \circ 1$ \\
\hline à å ç é è ê ë í ì î ĩ ñ ó ò ô ö & ä åéñ öü [ $\{[\}\}]$ á à â ä ã å \\
\hline PALLOS ANTIKVA & HUNGARUM ANTIKVA \\
\hline ABCDEFGH I J K L & A B C D E F G H I J K L M \\
\hline defghijklm nop & defghijklmnopqr \\
\hline
\end{tabular}

Figure 7. - Quatre fontes de Péter Csatai.
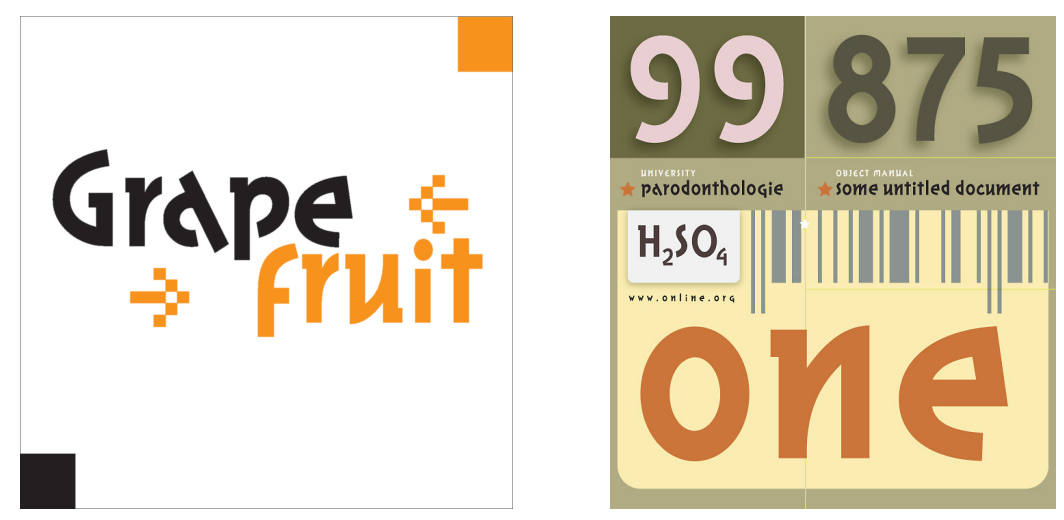

Figure 8. - Grapefruit d'Attila Győry.

crayon), artistique, fac-similé, etc. Nous ne pouvons présenter ici qu'une partie de leurs caractères (figures 11 à 15). Nous les avons choisis parmi les exemples envoyés par les dessinateurs eux-mêmes pour qu'ils soient présentés lors de la conférence de Brest. 
\{ S P E C I M E N 6

Characterum,

seu

Literarum,

$$
\text { Id eft : }
$$

Typorum Latinum

Probatifsimorum

Secundum suas Differentias

Exhibitorum,

\author{
qui \\ T Y R N A V I Æ \\ in Collegii Soc. Jesu
}

Officina Fusoria Formantur,

Atque pro Ornamento

Edendorum

Librorum

Ad proprium usum prostant.

\title{
Anno Domini MDCCLXXIII.
}

Figure 9. - Tyrnavia de Gábor Kóthay.

Fontes et dessinateurs de fontes hongrois contemporains 


\title{
ศБОUT DExÖDUS
}

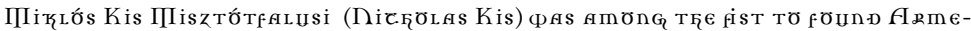

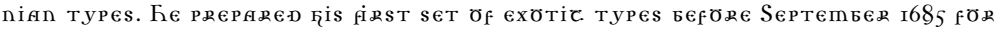

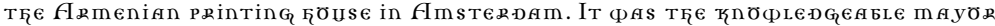

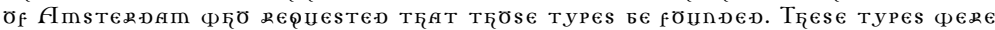

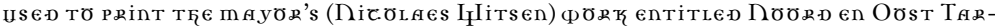

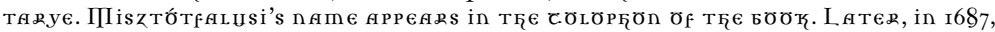

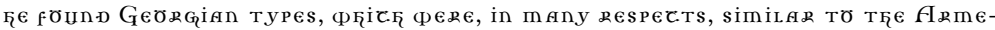

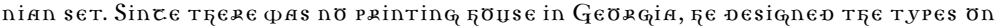

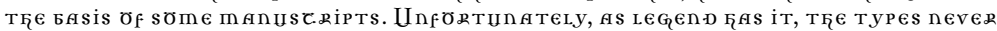

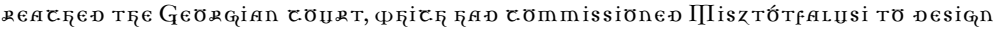

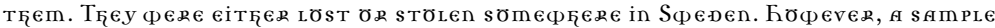

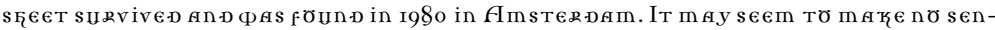

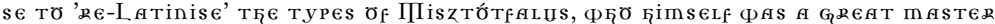

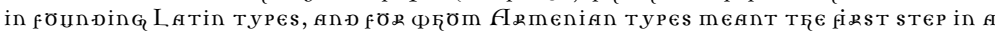
neџ Dizection.
\end{abstract}

Figure 10. - Nexodus d'Amondó Szegi.

Voici le nom de quelques unes de leurs créations, qui ne sont pas illustrées ici :

Gábor Kóthay :

— pour le texte : Alphabet, Archeype, Minerva, Moda, Tisza, Spirit, Zephyr ;

— scriptes : Anglia, Bacchus, Fizz, Zanzibar;

— spéciales : Arcade, Birdland, Depeche, Destijl, Faximile Flyers, Loop, Plexo, Totem, versus ;

— fontes spécifiques, cartes de vœux : Betabet, Bubble, Cats, Hungaria, Disasters, Subway, Surfing.

D'autres échantillons sont disponibles sur les sites : Fontana Type Foundry [8], Fontmunkások [9] et [12].

Amondó Szegi : Gehenna, Mantra, Telegdi, Nexodus (figure 10).

Heureusement, nous pourrons voir et utiliser de plus en plus d'excellentes fontes de ce genre dans le futur.

\section{La Place de TEX en Hongrie}

Avec Gábor Kóthay, nous avons commencé à parler de fontes libres de droits pour $\mathrm{T}_{\mathrm{E}} \mathrm{X}$. Le point de départ de cette discussion était que 


\section{円urePace}

A typeface design is, at its most basic level, a st You may still be asing the sane twentisix lett digits, but they are interpreted in a cinique way. typeface design might be likened to an original

Figure 11. - MuseFace d'Amondó Szegi.

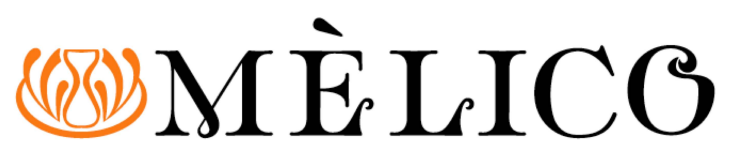

\section{MELGDIGSG.ARMGNIGSG.LIRICG. MUSICALE}

Figure 12. - Melico d'Amondó Szegi.

Glosso

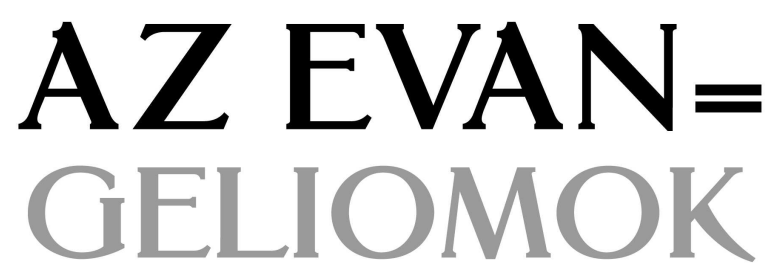

NAC, MELLYEKET VASARNA.

Figure 13. - Glosso d'Amondó Szegi.

Fontes et dessinateurs de fontes hongrois contemporains 
la Fontana Type Foundry en a de merveilleuses [8]. Gábor Kóthay et Amondó Szegi ont alors discuté avec Sun pour la version hongroise d'OpenOffice. Gábor Kóthay n'est pas contre l'idée de faire des fontes libres pour $\mathrm{T}_{\mathrm{E} X}$, et nous espérons pouvoir en reparler dans un futur proche.

Le premier style de document dessiné par des typographes hongrois est en construction. Il sera heureusement disponible pour les pages web de MATEX à la fin de cette année.

Enfin, la plupart des problèmes discutés dans [3] sont résolus, notamment le contrôle de la longueur de la dernière ligne d'un paragraphe et la division des mots contenant des traits d'union. Pour de plus amples détails, visitez les pages [13] ou [14].

Remerciements. - L'auteur est redevable aux dessinateurs mentionnés dans cet article et tient à remercier Péter Maczó et Yannis Haralambous pour leurs suggestions et leur aide. L'auteur remercie Jacques André de la magnifique traduction et pour la possibilité de mettre à jour cet article dans sa présente version.

\section{Bibliographie}

[1] Oszkár Bosкowitz. — Népfont Digital Type Foundry. http://www.nepfont.hu

[2] László BujákI. — Art Home - MûvészTábor. http://www.muvesztabor.hu

[3] Gyöngyi Bujdosó \& Ferenc WettL. — «On the localization of TEX in Hungary » ${ }^{3}$, TUG 2002 Conference proceedings, TUGboat 23(1), 2002, 21-26.

[4] Péter CsataI. - http://www.font.hu/digitart/csatai.html.

[5] Luc Devroye. - Type in Hungary. http://cgm.cs.mcgill.ca/ luc/hungary.html

[6] Nicholas Fabian. - The Hungarian Type designer Nicholas Kis. http://www.myfonts.com/person/kis/miklos/totfalusi/

[7] FontFont. — http://www.fontfont.com/fifteen/panels.

[8] Fontana Type Foundry, http://www.fontanatype.hu/frabout.html.

[9] Fontmunkások. — http://www.jobart.hu/fontmunkasok.html.

3. On pourra lire à ce sujet, des mêmes auteurs, «Adapter $\mathrm{T}_{\mathrm{E}} \mathrm{X}$ à la langue hongroise ", Cahiers GUTenberg no 44-45, novembre 2004, p. 115-127 (traduction française de Jean-Michel Hufflen). [N.d.T.]

Cahiers GUTenberg no 46-47-avril 2006 

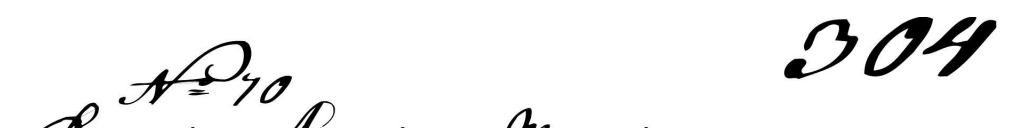

Breviex Antiqua Shatices pex......... 30. Gypous fushs pondexat .....

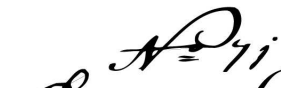

Breviex Cussiva flatices pex ........ 30." Gypors fusus pondexat ..... B... - 2i6.

Figure 14. - LaDanse de Gábor Kóthay.

\section{\%QUAMARINE \\ (2) \\ AaBbCcDdEeFfGgHh \\ $\mathrm{AaBbCcDdEeFfGgHh}$}

Figure 15. - Aquamarine de Gábor Kóthay.

Fontes et dessinateurs de fontes hongrois contemporains 


\section{Dessou}

Az uj anyag, az acel tette lehetove a ketlabas : jelentett elonyt, ahol jobb terkihasznalasra volt s: uleseken ulok labank helyre van szuksege. Ez a elkerulni, hogy a test a femhez erjen, ezert a cso : a karfajanal faval burkoltak. A hattamlat szov

Figure 16. - Dassau de Gábor Kóthay.

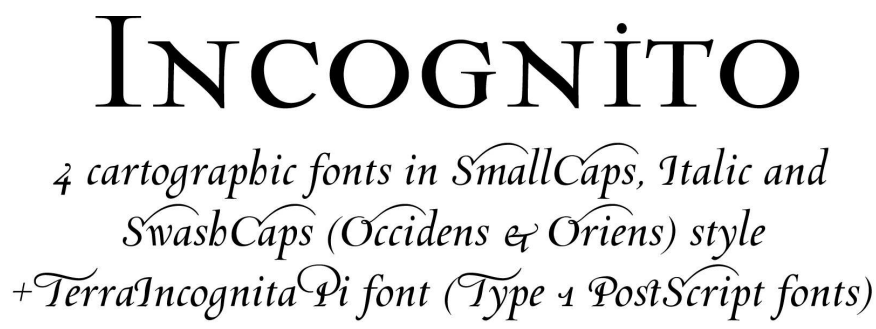

ÖKET AZ UR ISTEN PÉLDÁjÚl VETETTE MINDEN ISTENES ÉLETŰ EMBEREK ELEİBEN : MÉLTÓ-İS, HOGY AZ Ő PÉLDÁjOK FOGANATOSB LÉGYEN Mİ BENNÜNK, A' LELKİ JÓKBAN-VALÓ ELŐ-MENETELRE, HOGYSEM NÉMELY LANKADTAK TUNYASÁGA A' HÁTra MARADÁSRA. OH MELLy NAGY LELKİ

Figure 17. - Incognito de Gábor Kóthay.

Cahiers GUTenberg no 46-47 - avril 2006 
[10] FontShop. - http://fontshop.com/.

[11] Attila Győry. - Linotype.

http://www.linotype.com/7-653-7/gyoeriattila.html

[12] Hungarian type designers (Betűtervezők).

http://www.font.hu/digitart.html

[13] Hungarian LATEX home page, http://www.math.bme.hu/latex/.

[14] Hungarian $\mathrm{T}_{\mathrm{E}} \mathrm{X}$ Users Group (MAT $\mathrm{E}$ ), http://www.matexhu.org.

[15] In memoriam Tótfalusi Kis Miklós.

http://www.sk-szeged.hu/kiallitas/totfalusi/nyito.html

[16] Janson Text, Adobe Type Library,

http://www.adobe.com/type/browser/P/P_055.jhtml.

[17] Job Art Studio, http://www.jobart.hu/.

[18] Péter Maczó. — «Tótfalusi Kis Miklós myomdokain» (Dans les pas de Nicolas

Kis), Magyar Grafika, 2002(5), p. 2-7.

( Gyöngyi BujDosó

Department of Computer Graphics

and Library and Information Science

Institute of Informatics,

University of Debrecen

H-4010 Debrecen, P.O. Box 12 (Hongrie)

Fontes et dessinateurs de fontes hongrois contemporains 